\title{
Contingency Factors for Relationships in Complex Product Creation Environments
}

\author{
Donna Champion
}

\begin{abstract}
Current approaches to systems design and management are at the limits of applicability in modern complex product design environments. The collaborative nature of design activity is increasingly difficult to manage, where multi-disciplinary teams must share knowledge and co-ordinate the integration of technologies across different platforms and architectures. This paper describes a qualitative study to explore the critical factors in building and sustaining relationships across cross-functional teams in complex product creation environments. The study was undertaken in the Automotive sector, where market pressures demand swift integration of new technologies across platforms. A number of contingency factors have been identified and three strategic priorities for managers are suggested.
\end{abstract}

D. Champion $(\square)$

School of Business and Economics, Loughborough University,

Loughborough LE11 3TB, UK

e-mail: d.champion@lboro.ac.uk 